\title{
¿CUERPOS EXTRAÑOS EN EL MANAGEMENT URBANO? LA CONEXIÓN INSTITUCIONAL Y TEMÁTICA DEL PROGRAMA "SOZIALE STADT"1
}

Frank Eckardt ${ }^{2}$

Traducción de Alkos Cerda ${ }^{3}$ y Fernando Campos ${ }^{4}$

\section{El Programa "Soziale Stadt" en Alemania ${ }^{6}$}

Alemania se entiende como un Estado del Bienes$\operatorname{tar}$ (Sozialstaat), puesto que las necesidades básicas de las personas son garantizadas por el Estado cuando el ciudadano, por sí mismo, no puede hacerlo. Esta idea, la cual está consagrada en la Constitución alemana, ha llevado a que en los últimos

1 Traducción del texto Fremdkörper im städtischen Management? Die institutionelle und thematische Einbindung der "Soziale Stadt"-Projekte. En Greiffenhagen, Sylvia y Neller, Katja (Ed.) Praxis ohne Theorie? Wissenschaftliche Diskurse zum Bund-Länder-Programm Stadtteile mit besonderem Entwicklungsbedarf Die soziale Stadt. Wiesbaden, VS-Verlag, 2005.

2 Alemán. Profesor Doctor Habilitado, responsable de la cátedra de Sociología Urbana en la Bauhaus Universidad Weimar. frank. eckardt@uni-weimar.de

3 Traductor Español-Aleman. Estudiante de Medienkultur Bauhaus Universität Weimar treinta años muchos servicios sociales hayan sido garantizados, y de esta manera riesgos individuales como enfermedad, desempleo o accidentes no conduzcan a una crisis de subsistencia.

Sin embargo, en los últimos diez años la brecha entre pobres y ricos se ha incrementado, trayendo como consecuencia que muchas personas se viesen excluidas de espacios de socialización fundamentales, como son la educación y el trabajo.

4 Chileno. Sociólogo. Pontificia Universidad Católica de Chile. Máster en Vivienda de la Universidad Politécnica de Cataluña.

Sociología, Jena Universität y candidato a doctor en European Urbanism, Bauhaus Universität Weimar, Alemania.

5 [N de T] La traducción literal de "Soziale Stadt" es "Ciudad Social". En los casos en que dicho programa desarrolla documentos en inglés es nombrado también como "Ciudad Social" o bien "Ciudad Social e Integrativa". En esta traducción se ha preferido mantener el nombre del programa en alemán.

$6 \quad$ [N. de T.] Esta introducción ha sido especialmente escrita por el autor para la traducción de su artículo al castellano. 
Desde los años noventa la preocupación por dicha situación en Alemania ha ido en aumento, puesto que esta exclusión podría llevar a que, en determinadas ciudades, la insatisfacción de la población desembocase en violencia. Esta es la razón por la cual tres Estados Federales ${ }^{7}$ comenzaron con importantes programas de política pública decididos a apoyar a ciertas partes de la ciudad (Stadtteile) que presentaban una alta concentración de problemas sociales. En el año 1999 este enfoque fue adoptado para toda Alemania y paulatinamente implementado en más de doscientas ciudades.

El "Soziale Stadt” es un programa de los Estados Federales Alemanes (Bund-Länder-Programm), en el cual el gobierno nacional, el gobierno federal y las ciudades respectivas trabajan interrelacionadamente, debiendo cada uno de ellos aportar un tercio de los costos totales de la intervención. Producto de la estructura federal de Alemania, el gobierno nacional no puede mezclarse en los asuntos locales, y por consiguiente está impedido de financiar directamente proyectos de regeneración barrial, algo similar al caso francés. Las intervenciones que las ciudades asumen en ámbitos como desempleo y pobreza son reembolsadas a través de un sistema nacional, no obstante esto no opera para prestaciones y servicios adicionales, necesarios en ciudades con altas tasas de cesantía.

7 [N de T] Los tres Estados Federales señalados son: NordrheinWestfalen, Bremen y Hamburgo

188 revista invi № 72 / Agosto 2011 / Volumen № 26: 187-205
En el contexto de un Estado del Bienestar como el Alemán, históricamente se ha excluido la posibilidad de que ciertas ciudades e individuos sean protegidos más que otros. Por el contrario, el programa "Soziale Stadt" hace posible exigir una atención especial a barrios deprimidos de la ciudad, donde aún cuando el programa es entendido y motivado desde su dimensión "social", es implementado y concebido desde un ministerio focalizado en el desarrollo urbano. Esto ciertamente trae desventajas, como por ejemplo que la competencia principal en términos de desarrollo urbano se encuentra a nivel del Estado Federal, lo que sin duda debe ser considerado. En otras palabras, es necesario articular diferentes niveles del gobierno para que contribuyan financieramente.

En resumen, después de más de diez años de existencia del programa "Soziale Stadt" el balance es destacable, lo que se puede afirmar ya que en efecto en las ciudades alemanas no se dieron las violentas protestas que tuvieron lugar en Francia en el año 2005. Esto no debe impedir una visión crítica del asunto, ya que a pesar de las intervenciones del "Soziale Stadt" la situación de muchas personas en la última década es considerablemente peor que antes; en base a reportes oficiales del gobierno, hoy en día casi un millón de personas más viven bajo condición de relativa de pobreza. En cada ciudad 
se dan situaciones contradictorias; por un lado a través del "Soziale Stadt" se ha logrado muchísimo y de a poco se observan cambios, pero por el otro lado no se han cumplido ciertas expectativas existentes referidas a combatir la fragmentación social o fortalecer la economía local.

Para muchas personas el "Soziale Stadt" ha significado un verdadero apoyo en su vida de barrio, y esta experiencia ha enriquecido la perspectiva de cómo los vecinos pueden entre ellos mismos ayudarse. Estas dinámicas son fundamentales, sobre todo en un momento donde muchas personas en Alemania y en todo el mundo se ven afectadas y confundidas por la crisis mundial. Investigaciones en el contexto del "Soziale Stadt" muestran, reiteradamente, que las personas con menores posibilidades sociales, financieras y culturales necesitan directamente del barrio para poder orientarse en el día a día y planificar su futuro. Esto es posible en gran medida gracias al "Soziale Stadt", que ha desplegado iniciativas para grupos específicos como niños, jóvenes, mujeres, adultos mayores y extranjeros.

Comúnmente, una de las principales peticiones para el "Soziale Stadt" desde el barrio es que se construya un centro comunitario o de reuniones, donde el encuentro no sea obligatoriamente para "tomar el té", ya que para muchas personas este centro puede ser el único lugar donde ir fuera de la propia casa. Si bien problemas estructurales como el desempleo no han sido superados, para Alemania la activa- ción del barrio es una importante innovación social, la cual es valorable de ser observada y discutida sobre la base de la experiencia latinoamericana.

Ideas como el "presupesto participativo" fueron tomadas desde Brasil, mientras que otros ejemplos respecto a "organización comunitaria" y "empoderamiento" fueron inspirados en el caso norteamericano. En el contexto alemán -y principalmente desde la perspectiva de un Estado del Bienestar que se reduce-, estos enfoques inicialmente resultaron muy extraños, pues muchas facultades del Estado han sido limitadas.

Cómo los ciudadanos pueden y deben comprometerse con su barrio fue una nueva experiencia para Alemania, y trajo consigo una nueva comprensión sobre el Estado, la Ciudad y el significado de ser ciudadano. Observar y discutir en conjunto sobre la experiencia Alemana es seguramente para los académicos, profesionales y la sociedad civil latinoamericana un gran desafío al cual quedan todos invitados.

\section{Introducción}

Luego de la reforma de la administración dentro del marco del "New Public Management" (NPM), que fue intensamente discutida, probada e implementada en Alemania, quedó un cierto grado de desencanto respecto a las oportunidades y posibi- 
lidades de encontrar en ella un particular cambio de enfoque ${ }^{8}$. De acuerdo con la opinión de muchos observadores, existe en esta experiencia no solo un "problema de implementación", sino también un "problema de concepción". Las principales dificultades que se hacen visibles con el NPM se pueden dividir en cuatro puntos. Primero, existe una tendencia general a considerar la reforma fundamentalmente como algo positivo y finalizado. En segundo lugar se admite que las reformas inspiradas por el NPM conllevan dificultades, sin embargo estas se tienen bajo control. De esta manera, tanto académicos como políticos exigen que la implementación del NPM sea completa, eliminando cualquier contradicción a nivel local. En tercer lugar existen dudas, las cuales apuntan a que si en efecto en el NPM todo fuese correcto, serían entonces necesarias modificaciones también al marco teórico de la reforma administrativa. Y en cuarto lugar está en boga una perspectiva relativamente nueva de la reforma institucional, la cual junto al concepto de "gobernanza" se esfuerza por una integración del NPM en el contexto de las continuas transformaciones a la política local. Por otro lado existen dificultades, como por ejemplo la crisis financiera comunal, que son provocadas externamente y no están en ninguna vinculación con reformas en las relaciones administrativas. Finalmente, existen aquellas tendencias que no quie-

8 NASCHOLD YBOGUMIL, 2000.

9 MINGUE et al., 1998. ren saber nada de reformas. Esas afirmaciones son para tomarlas en serio, pues podrían ser una señal del distanciamiento entre la teoría y la práctica.

En lo que sigue se discutirá la idea de si con el prometedor concepto de "gobernanza" es posible una adecuada teorización de la práctica ${ }^{10}$. Esto conlleva problemas fundamentales, ya que el término "gobernanza" es un concepto activamente aplicado y desarrollado, tanto normativa como analíticamente. Es importante destacar que la concepción política de "gobernanza", o bien de "buena gobernanza", no se puede utilizar para la reflexión teórica; es más, surge la pregunta de hasta qué punto dichas ideas -abstractas- encuentran entradas en las subáreas de la práctica política. La teoría de la gobernanza parte la base que, como mínimo una parte de esta ya se ha implementado. En efecto, la investigación internacional sobre la teoría de la gobernanza remite gran parte de su empiria a casos de "buena gobernanza" en países en desarrollo.

Solo en los últimos años el concepto de gobernanza cobró relevancia para el programa "Soziale Stadt" y para la política comunal europea en general. En este contexto, un impulso no despreciable fue causado por el "White Paper on Urban Governance" de la comisión Europea ${ }^{11}$, el cual no solo alude a una filosofía de diseño para programas individuales (como por ejemplo URBAN), sino que

10 BOGASON, 2000.

11 ATKINSON y ECKARDT, 2003. 
también propone una definición fundamental de política comunal, algo así como ha sido la idea ampliamente aceptada en los años noventa de privatización de los servicios comunales.

\section{Gobernanza y Soziale Stadt}

Hoy en día falta en la opinión pública en general una reflexión concienzuda a la pregunta de cómo se congenia el concepto de "gobernanza" con los problemas del "Soziale Stadt". Los enfoques de gobernanza constituyen una estrategia de reforma para la política comunal, la cual trasciende los cambios institucionales llevados a cabo hasta ahora en el marco del NPM. Nosotros sostenemos que la fallida recepción tanto de las reformas del NPM llevadas a cabo hasta ahora, como también su futura compatibilidad con la estrategia de reforma de la gobernanza de la Comisión Europea, constituyen en conjunto un considerable déficit con respecto a los efectos que pudiera tener este programa. Puesto que una política de integración social siempre se debe entender como una tarea de toda la ciudad, surge como imprescindible una reflexión acerca de la relación entre "Soziale Stadt" y gobernanza local12.

Existen muchos puntos de encuentro entre el programa federal "Soziale Stadt" (Difu 2000, 2002, Walther 2002) ${ }^{13}$ y la teoría de gobernanza:

12 LAHNER Y ZIMMERMANN, 2005.

13 DIFU, 2000. DIFU, 2002. WALTHER, 2002.
- Las ciudades participantes y las municipalidades son invitadas a tener una actitud pro-activa contra la fragmentación social.

- Las ciudades, en un aspecto político de red, deben posibilitar la responsabilización, la cual conllevará en la cooperación de los Estados Federales y sus Ministerios a una fuerte contribución para la cohesión social.

- Losenfoques del “Soziale Stadt”, así como los de la gobernanza local, enfatizan la importancia de la participación ciudadana y del "capital social"14.

Los teóricos de la gobernanza creen fuertemente en la importancia de las tecnologías de la información y de la comunicación, y ven en ellas nuevas oportunidades para el desarrollo de la ciudad. Sin negar las diferencias gravitantes entre los enfoques de gobernanza local y la programática del "Soziale Stadt", en este contexto predomina una fuerte coherencia, pues ambas:

- Destacan las redes con un alto número de actores públicos de diferentes reparticiones.

- Las relaciones institucionales entre dichos actores públicos se alinean en función de los objetivos de la política local.

- Las dos buscan confianza y transparencia en los procesos de decisión urbanos, a través del

14 WENDLAND, 2002. 
fortalecimiento de las políticas de participación ciudadana.

De este modo, se plantea la pregunta "ihasta qué punto el programa "Soziale Stadt", con sus exigencias de estructuras orientadas administrativamente (Grupo de dirección y el management del barrio), ${ }^{15}$ ha creado prácticas para que esta reforma administrativa impulsada exteriormente sea en efecto más que un intermezzo ficticio?” ¿O es simplemente un factor perturbador en una estructura organizacional que sigue su propia lógica autopoyética? La gobernanza local en su idea principal apunta a tres niveles de la estructura política: i) lograr una transformación duradera del desarrollo político comunal; ii) dónde el foco se dirija a la conducción de procesos locales; y iii) que la decisiva innova-

15 [N de T] El programa "Soziale Stadt" diferencia entre Quartiermanagement, que es la instancia de coordinación de los proyectos en el barrio, donde se da la posibilidad de que un conjunto de actores sociales-organizados y no organizados-participen de la toma de decisiones, y el Quartiermanager, que es el profesional responsable de los proyectos del "Soziale Stadt" en el barrio y que a su vez coordina al Quartiermanagment. En lo que sigue se traducirá este último concepto como "management del barrio". El programa "Soziale Stadt"a su vez define al Steuerungsgruppe, que como su traducción lo señala es el grupo de dirección. Esta instancia incorpora obviamente al Quartiermanager (profesional responsable del barrio), pero esta vez en conjunto con los representantes de las diferentes reparticiones de la ciudad con interés en el barrio, como pueden ser: juventud, empleo, educación, obras públicas, etc. Este grupo de dirección se encargará de establecer la comunicación entre los actores sociales y las autoridades políticas, ellos priorizan y discuten las actividades o proyectos dentro del barrio. ción sea el alineamiento de los actores sociales respecto de la totalidad de la sociedad urbana. Es por esto que en lo que sigue se debe discutir si dichos tres niveles: i) sustentabilidad, ii) dirección y iii) alineamiento externo, están presentes en el Programa "Soziale Stadt" y concuerdan o no, con los objetivos de la "gobernanza Local".

\section{Alineamiento Externo (Externe Ausrichtung)}

Tanto en el marco de una entrevista ${ }^{16}$ a los responsables del proyecto en todos los niveles, considerando especialmente al Management del barrio y las oficinas internas de coordinación, así como también, desde una mirada superficial, a la ejecu-

16 Las experiencias aquí expuestas se basan en entrevistas escritas semi-estructuradas con diferentes actores de los proyectos "Soziale Stadt" en Hessen y entrevistas profundas, las cuales fueron realizadas en el marco de los estudios del proyecto "Soziale Stadt" entre el año 2002 y 2004 . El número de encuestas realizadas es de 24 . 
ción del programa en otras ciudades, se observa una asombrosa diversidad de temas y acciones realizadas a través del programa. Esto, en términos del enfoque sobre gobernanza, se comprende como una contribución al "Construcción de Comunidad" ("Community Building"), ya que diferentes intereses locales y necesidades pueden expresarse y posteriormente pueden llegar a ser considerados de acuerdo a su mérito. Es sin duda un efecto colateral positivo del programa el hecho de que una gran variedad de acciones sean apoyadas financieramente. Sin embargo, surge la pregunta de si esto tiene sentido en la concreción de proyectos tan diferentes como la renovación de una plaza de deportes o la decoración de una casa para el adulto mayor. En este sentido, iofrece la institucionalización del "grupo de dirección" y el "management del barrio" suficiente flexibilidad y potencial de dirección?

En primer lugar se confirma la idea de que con la implementación del programa en cada territorio, la diversidad de acciones no tiene la intención de respaldar un punto de vista prioritario en la selección de un aspecto único para el desarrollo de la ciudad. La selección de los proyectos específicos y de los barrios está sujeta más que nada a un procedimiento inconsistente, el cual la mayoría de las veces depende únicamente de una decisión legitimada por los correspondientes consejos municipales (Stadtrat); sin embargo, más que esta aprobación formal debería ser de interés para futu- ras apreciaciones el proceso de discusión anterior a dicha instancia, el cual será en definitiva el que organizará la toma de decisión.

Con respecto a lo anterior, existieron diferentes puntos de partida en las ciudades seleccionadas. Hubo, por ejemplo en algunos casos, una fase en la preparación de la decisión fuertemente orientada por las personas y el contexto social. Por contexto social se entiende aquí que ya estaba presente una red de actores establecidos, los cuales fueron contactados de manera nueva o bien reclutados desde redes antiguas, con el fin de iniciar la propuesta en el consejo municipal y todas las medidas iniciales necesarias. Resulta decisivo en este proceso el anclaje de los proyectos de intervención barrial al quehacer de los partidos políticos, así como también la referencia a una política comunal con la potestad formal como informal de imposición, la cual sea ejecutada a través de personas autorizadas. Al mismo tiempo, se da cuenta que en casos puntuales fue necesario algo así como un representante simbólico de la sociedad civil o del vecindario, el cual debiese comunicar una posición no-partidista. Es por esta vinculación de diferentes actores que al inicio de los proyectos del "Soziale Stadt" se genera una situación que puede llevar a un cierto grado de apertura de la política y de la administración política comunal, lo cual enriquecería la rutina de la administración en lo que refiere a la política social. 
En comparación con las discusiones teóricas de la "gobernanza local", la situación inicial del proyecto "Soziale Stadt" se presenta como evidentemente débil. En este contexto, la necesidad de una política social estatal que elija uno, o como máximo dos proyectos de barrio, cubre la pregunta por la legitimación, a saber, de tipo formal, pero ella no se basa en los enfoques de gobernanza, los cuales refuerzan las decisiones tomadas por actores colectivos, a saber la "Ciudad"17. En términos de la selección de los barrios se observan diferentes situaciones. Por un lado se observa un efecto de envidia de otros sectores de la ciudad no seleccionados y una clara arbitrariedad en la elección de futuros proyectos. En ciertos casos, para que la ciudad no perdiera sus posibles inversores, la elección de los barrios fue establecida tomando en consideración solo las proposiciones e ideas hechas por actores previamente establecidos. No obstante lo anterior, existen situaciones en las que se generaron procesos largos y duraderos de discusión de los distintos actores que permitieron una búsqueda intensiva de las propuestas de implementación adecuadas. En este proceso fueron elegidas ideas de proyectos de acuerdo con la imagen y la representación exis-

17 [N de T] El argumento de la Ciudad como "actor colectivo" es tomado del ensayo de Max Weber que lleva el mismo nombre. En español ver: WEBER, Max. La ciudad. 1987. Madrid, Las Ediciones de la Piqueta.

194 revista invi № 72 / Agosto 2011 / Volumen № 26: 187-205 tente del barrio, de acuerdo a la noción de "area based"18.

Dentro del proceso de selección de los barrios se presenta como una problemática creciente que su caracterización responda de manera exclusiva a recursos del sentido común, como por ejemplo "dónde en la ciudad se encuentran los puntos más críticos”. En la opinión de muchos entrevistados, la falta de integración de actores no-establecidos o no-representados y la apertura comúnmente solo marginal del debate en la selección de los barrios, amenaza con iniciar o contribuir a un proceso de estigmatización del "Soziale Stadt". Se critica también, desde una perspectiva basada en los distritos de la ciudad, que la elección de un área determinada no esté siempre justificada. Aquí se señala como ejemplo que existen necesidades iguales o mayores a las de las zonas seleccionadas en un nivel micro, como puede ser un bloque de casas dentro de las zonas no incluidas en el programa.

Si la decisión tomada en cada barrio favorecido con el programa "Soziale Stadt" fue adecuada, es la pregunta general que se plantea en este contexto. El enfoque del programa ha resultado problemático también porque recurre a la percepción ya exis-

18 [N de T] La aproximación basada en el área (area based approach) refiere a cuando el territorio es usado como unidad de intervención sobre problemáticas socio-espaciales, en oposición a una aproximación basada exclusivamente en los grupos sociales con independencia del territorio en que ellos se localizan.

OPINIÓN: ¿Cuerpos Extraños en el Management Urbano? La Conexión Institucional y Temática del Programa "Soziale Stadt" / Fernando Campos 
tente de los diferentes barrios de la ciudad, y en especial a cómo se han ejecutado los recursos y las medidas de inversión de otros programas de política pública. La necesidad de evaluar aspectos de eficacia del programa no ha sido considerada; por ejemplo cabe la pregunta de si "en otros lugares de la ciudad se podrían lograr mejores resultados con la inversión, incluso cuando allí la situación aún no es tan terrible". Un debate público y una red de actores transparente podrían actuar aquí en cierto modo como un corrector y también como una instancia de control.

De esta forma algunos enfoques de las ciencias sociales son cuestionados, en gran parte pues quieren plantearse como aptos para identificar los espacios de la "fragmentación social" sólo a través de una categorización cuantitativa y residual de los barrios. Resulta importante además, en opinión de los entrevistados, que en el sentido de "Construcción de Comunidad" ("Community Building") la fase de preparación no ha sido suficientemente utilizada para hacer del programa "Soziale Stadt" una política de identificación de los ciudadanos. Las dificultades anteriormente mencionadas, como la apertura de las redes del programa a actores externos y la posibilidad de congeniarla con la voluntad de los tomadores de decisiones comunales (decision-makers) parecen ser determinantes rumbo a la iniciación de los proyectos. Un fuerte indicio del bajo anclaje de la programática del "Soziale Stadt" en la política comunal es la escasa referencia a con- ceptos de desarrollo urbano de primer orden, los cuales han sido propuestos en los últimos años casi en la totalidad de las comunas de Alemania de manera superficial y con referencia sólo al desarrollo de modelos de ciudad. Esto también es aplicable al proceso de la "Agenda Local 21", el cual es vinculado sólo de manera escasa y nominativa.

\section{Dirección (Steuerung)}

Se debe reconocer como una importante innovación del programa "Soziale Stadt" que su dirección fue planeada, y esto se debe considerar como un signo de la sustentabilidad de su programática. De hecho, los resultados confirman que las ciudades se vinculan y comparten entre sí respecto de la conducción de los proyectos fuera del marco de las responsabilidades dadas. Es por esto que resulta difícil, en los casos aislados, hacer evaluaciones de los resultados de este intercambio. Las personas entrevistadas que participan en el programa se han negado, en gran parte, a emitir juicios respecto de sus propias actividades de management, siendo las visiones personales en este contexto difíciles de evaluar. Declaraciones respecto de las "buenas" o "deficientes" prácticas reflejan entramados de poder, jerarquías y competencias en una escala micro, las cuales pueden conducir a la exteriorización de diferentes posiciones de partida de los grupos directivos a nivel interdepartamental, o mejor dicho, a nivel general de las oficinas del grupo de dirección. 
Por último, existen muchos signos que no se encuentran en la experiencia concreta del trabajo en conjunto, los que han traído evaluaciones negativas.

Sorprendentemente existe una gran cantidad de juicios positivos y de razones para una evaluación positiva de la cooperación inter-administrativa. Entre otras cosas, se sostiene que es un alivio para la ejecución del proyecto tener con los colegas un intercambio estructurado, o bien, en el sentido de las preocupaciones, se ha argumentado que a través de los grupos directivos se pueden abordar adecuadamente los problemas de los proyectos a corto plazo. Las preguntas concernientes a cuál es el estatus del grupo de dirección en relación con las decisiones de primer orden sobre aspectos esenciales del proyecto fueron respondidas con evasivas, como por ejemplo la entrega de permisos de construcción. En estas preguntas da la impresión que la oficina municipal en cuestión considera a menudo la votación del grupo de dirección como un mero trámite. Mientras más esto ocurra, más a menudo la importancia del grupo de dirección será cuestionada. Las reuniones de los grupos de dirección en su mayoría se programan "en caso de necesidad", lo que supone describir la situación evaluada -en el momento de la reunión- como algo problemático. Excepciones como en Wetzlar ${ }^{19}$ confirman la

19 [N de T] Para mayor información sobre este barrio se puede visitar el sitio web del programa "Soziale Stadt":

http://www.sozialestadt.de/gebiete/gebietAnzeige.php?id=86 posibilidad de que el grupo directivo puede tener fuerza en la perspectiva de gobernanza, a través de reuniones periódicas, amplitud temática y la inclusión de actores no gubernamentales.

Las diferencias encontradas en la evaluación del grupo de dirección se pueden interpretar de muchas maneras. Cuando se trata de la evaluación general de su funcionamiento pareciera no ser relevante en qué posición o en qué oficina se encuentra la ubicación del grupo de dirección, como tampoco parece marcar diferencia la asignación de los proyectos a ciertas oficinas. Por el contrario, resultan claras las diferencias personales en los estilos y expectativas de cooperación. Esto tiene que ver en parte con los antecedentes profesionales de los participantes en la dirección, pero también con la posición jerárquica de los entrevistados. Una fuerte cooperación es principalmente deseada por las instituciones u oficinas sectoriales más débiles, como por ejemplo sociales, juveniles y culturales.

La posición relativamente débil del grupo de dirección en la ejecución del proyecto se debe en parte a su conexión con la totalidad de los procesos administrativos, los cuales fueron evaluados por los actores de forma dispar. En una escala de 1 a 10 (donde 1 es poco/malo y 10 es mucho/muy bueno) hubo claramente evaluaciones tanto muy positivas (10), 
como evaluaciones más bien moderadas (3). En promedio, aunque con una gran dispersión, existió una evaluación positiva del programa $(5,7)$. Aquel resultado está relativizado por la pregunta acerca del grado supuesto de conocimiento o familiaridad con los proyectos del "Soziale Stadt" dentro de la propia administración. Este conocimiento o familiaridad tuvo un valor medio calculado más bajo $(4,3)$ y con una menor dispersión. Aquellos que están convencidos del buen funcionamiento del grupo de dirección aprecian también de manera alta -positiva-su notoriedad al interior de la institucionalidad. No obstante, ellos consideran que dicha notoriedad está bajo el nivel de funcionamiento esperado para el grupo de dirección. En otras palabras, a pesar de que los proyectos "Soziale Stadt" son poco conocidos, los funcionarios responsables observan la conexión con la administración comunal como medianamente exitosas. Sería diferente responder a la pregunta de si los gremios políticos locales conocen efectivamente los proyectos "Soziale Stadt", pues en parte se asume que los políticos conocen los proyectos antes que la administración, no obstante otros entrevistados que participan del programa opinan lo contrario. Para estas diferentes evaluaciones no hay una razón evidente. Aunque el conocimiento de los políticos se encuentra, en promedio, ligeramente por sobre el de los administrativos, no alcanza un valor que justifique el juicio de una positiva relación con la administración comunal.
La dirección de los barrios dentro del proyecto "Soziale Stadt" es vista principalmente como un procedimiento al interior de la institucionalidad, el cual parece corresponder en su forma actual a un requisito para los funcionarios sectoriales competentes, siempre y cuando este no sea utilizado "como una demanda" sobre ellos. A la institución y a la política se le impugna un bajo conocimiento de los proyectos, lo que indica que la orientación hacia el resto de la institucionalidad pública del grupo de dirección parece ser poco relevante. De otra manera, el desconocimiento autopercibido de las intervenciones del "Soziale Stadt" por colegas y actores políticos hubiera sido excluido de una evaluación positiva en la integración comunal.

Un signo que supone una transición desde la teoría de gobierno local hacia la teoría de gobernanza sería la participación de actores externos en la dirección del programa. Estos actores no son previstos a nivel del grupo de dirección, por el contrario, se introduce un nivel adicional donde ello ocurre, el management del barrio ${ }^{20}$. En esta instancia, según los encuestados, pueden participar una gran cantidad de diferentes grupos sociales e individuos y de maneras distintas en el trabajo concreto de los proyectos. Los encargados de los barrios (Quartiersmanagern) entregaron una descripción detallada de este modo de participación, el cual es resultado en su mayoría de la formulación de tareas, las que

20 BERNT Y PRITSCHE, 2005 
prevén de manera general la activación del barrio, la participación de los actores, el desarrollo de proyectos de ayuda a la vecindad y la actividad social y local. El management del barrio no es en cada caso equiparable a la dirección de los proyectos en el barrio, puesto que este se puede comprender en algunos casos como una parte del proyecto, y entonces como un elemento externo de dirección. Si en principio no se prohíbe una dirección interna, o mejor dicho autónoma, se plantea la pregunta, ¿por qué se establece una dirección de proyecto a nivel de barrio al lado del management del barrio?

La comunicación entre las oficinas vinculadas al grupo de dirección y el nivel del management del barrio orientado al vecindario no están uniformemente reguladas, y se concretizan de maneras muy diversas, desde relaciones muy intensas a otras meramente formales. En un caso ideal, como lo es Frankfurt Unterliederbach Ost ${ }^{21}$, existe una reunión de coordinación del proyecto en la cual, bajo la dirección de la Oficina de Planificación Municipal (o Dirección de Urbanismo Municipal), participan el management del barrio y la dirección de proyectos en el barrio. A partir de la estructura organizativa, en conexión con el Programa "Soziale Stadt" podría describirse el caso anterior -Frankfurt Unterliederbach Ost- como una "buena

21 [N de T] Para mayor información sobre este barrio se puede visitar el sitio web original del programa "Soziale Stadt": http:// www.sozialestadt.de/gebiete/gebietAnzeige.php?id=75 mezcla" pues supone aproximaciones de Bottom-Up y Top-Down ${ }^{22}$. En la práctica se pueden escasamente señalar formas reguladas de información, y aún menos formas reguladas de decisión; ellas en efecto, median entre ambos niveles: "grupo de dirección en el barrio" y "management del barrio /dirección de proyecto". Si en el análisis ejemplos tales como los que dan cuenta de una conexión funcional en dichos niveles fuesen incluidos, por lo menos se cumplirían las condiciones de comunicación para una mayor coherencia de las actividades y de los objetivos.

En este sentido, la programática de "Soziale Stadt" hace posible la creación de redes ${ }^{23}$ entre la administración y los actores sociales, pero ¿de qué manera el enfoque de redes funciona y qué resultados entrega? Se mantiene como una incógnita, debido a la duración cortoplacista de muchos proyectos. Da la impresión a primera vista que el management diario se realiza en la parte inferior y la dirección en la parte superior; esto debiera considerarse como un problema cuando los dos niveles no están eficiente y estrechamente conectados. En caso que esta precondición se cumpliese, aún así no estaría siempre satisfecha la idea de participación en un sentido maximalista. Los ejemplos examinados muestran que los resultados medidos en la aplicación de la idea de Bottom-Up, resultaron ser más

22 SCHADER-STIFTUNG, 2001.

23 BOIVARD et al., 2002 
impresionantes cuando los niveles altos, grupo de dirección, fueron concebidos fuertes en decisión. Esto resulta claro, por ejemplo cuando la jefa de asuntos sociales de Kassel ${ }^{24}$ toma parte como motor de conducción en este gremio. Esta observación contradice tanto una filosofía de diseño orientada a la participación como también al enfoque de redes, en dónde las decisiones son manejadas en primera línea desde arriba y desde adentro hacia fuera. En contraposición con la doctrina pura de la sociedad de redes (Networking Society), los teóricos de las gobernanza como Patrick Le Galès destacan que esto se trata de una transformación en el rol de los políticos a nivel local, quienes utilizan sus potencialidades y sus capacidades de decisión en el sentido de un "Bonum Commune" (bien común) formulado en las redes de gobernanza. En este contexto podría explicarse la supuesta contradicción entre la utilización simultánea de la idea intensiva de redes y un nuevo rol de los políticos locales.

\section{Sustentabilidad (Nachhaltigkeit)}

Los aspectos administrativamente relevantes de las ciudades en el programa "Soziale Stadt" no solo deberían tener como finalidad la distribución de las

24 Kassel es una ciudad ubicada en el norte del Estado Federal Alemán de Hessen, mismo Estado en el que se localiza Frankfurt am Main inversiones federales, sino también entender estas como una oportunidad de promoción del apoyo a largo plazo del capital social de las ciudades. En este sentido, es imprescindible no solo discutir acerca de la sustentabilidad del programa en base a los proyectos, sino también tener en mente la estructura relativa a la institución. Las medidas pueden significar una revalorización de la conducción de los procesos sociales. Por lo tanto, este enfoque podría ser entendido como una contribución a la modernización de la administración pasando desde un rol de distribuidor a un rol de moderador.

Esta interpretación lleva a suposiciones como que las redes iniciadas y desarrolladas dependerán de la fecha de caducidad de la de la inversión federal (Bundesförderung); en este sentido las percepciones de los actores entrevistados resultan optimistas. Este optimismo radica, por un lado, en el positivo trabajo en conjunto, y por otro lado en el positivo efecto del proyecto. Como condición previa para un desarrollo sustentable de las intervenciones estatales, en primer lugar, se debe apuntar a la pregunta de si también las medidas planeadas para el barrio y su correspondiente grupo objetivo son adecuadas. Sería muy apresurado aquí preguntarse por juicios definitivos, puesto que también se dan dificultades, sobre todo en los tomadores de 
decisiones comunales, los cuales entregan respuestas que no resultan ser estratégicas. En referencia a la perspectiva de largo plazo, la cual supone un enfoque sustentable de la programática del "Soziale Stadt", serán tomados los juicios iniciales solo como una evaluación de proceso en conexión con una evaluación de productos.

Si bien resulta difícil, por ejemplo, estimar si un centro comunitario en construcción luego de su finalización será sustentablemente utilizado, en los debates comúnmente se pone énfasis en un desarrollo sustentable de la ciudad, donde la administración debe proporcionar las condiciones generales que permitan su uso en el largo plazo. Esta exigencia define a la administración comunal como una autoridad prestadora de servicios, la que con sus instrumentos permitiría el trabajo preparativo, sin el cual los proyectos del "Soziale Stadt" finalmente no podrían salir a la luz. Se plantea entonces la pregunta, ien qué sentido el management del barrio y el grupo de dirección pueden llegar a ser elementos para un desarrollo socialmente orientado de la ciudad? En nuestras entrevistas se aclaró en este sentido que la distribución de tareas adicionales, en el marco del grupo de dirección, es aceptable para los trabajadores y las oficinas participantes siempre y cuando el trabajo extra-obligatorio sea acordado previamente por las partes, donde al menos se aborde el intento por redes más amplias, estando siempre presente la esperanza de que el grupo de dirección a largo plazo se reúna menos y a través de la ayuda de actores externos se pueda hacer más fuerte. Se ha de agradecer, en este sentido, el alto grado de compromiso de los funcionarios, los cuales dan la fuerza adicional al grupo directivo. No obstante el hecho que yo no considere esta situación como duradera, es para mi propia comprensión y supone por consiguiente que el "Soziale Stadt" puede llegar a ser un factor perturbador cuando no se integre de un modo distinto dentro de la administración comunal.

Siempre y cuando se tomen medidas infraestructurales por parte de la comuna en el marco del programa "Soziale Stadt", el consejo municipal, o como mínimo las oficinas competentes, procurarán una conexión a largo plazo en las estructuras administrativas comunales, o mejor dicho, seguirán asegurando esta. Así se presentaron típicamente la construcción y la fase de puesta en marcha de planes de inversión en el período de ejecución del programa, garantizando la continuidad de los proyectos; algo así como asumir la administración a través de una asociación de apoyo, la cual debe coordinar, como ya se señaló, la administración, el funcionamiento y el dialogo. De tal manera se desarrollará un marco organizativo, el cual promete una perspectiva sustentable para los proyectos particulares, según la composición de la red y su apoyo a la política comunal. Algo similar puede decirse de los proyectos orientados comunalmente, los cuales con frecuencia organizan a un grupo igual de organismos 
responsables para el período posterior a la financiación inicial.

Estos preparativos organizacionales no permiten, evidentemente, hacer un juicio acerca de las posibilidades reales de continuidad de los proyectos individuales luego del término del programa federal. En las entrevistas se dio casi por unanimidad que la reforma de las finanzas comunales y la dotación de financiamiento local, ambas en conjunto, serán probablemente los factores más importantes que determinarán la continuidad de cada proyecto de manera individual. Respecto de este problema, en las entrevistas el ítem "la continuidad deseada del programa federal" recibió la más alta aprobación en comparación a todos los temas preguntados $(8,7)$. Las necesidades de ejecución adicionales en cada una de las ciudades para apoyar sus proyectos propios, son en principio reconocidas y se expresa como un deseo que futuros proyectos debiesen venir con la bendición de un subsidio estatal o comunal. Esto significaría una aprobación de contenidos del programa por los actores comunales, la cual solo es relativizada a través de unas pocas declaraciones en otros contextos.

La dependencia de la financiación comunal y el extremo respaldo externo a través del gobierno federal disminuye de forma plausible la confianza que existe en relación a la sustentabilidad del proyecto en su conjunto. De seguro estas son las principales preocupaciones planteadas por los actores, e inclu- so es indudable que el desarrollo social depende en gran parte del desarrollo económico. Pero entonces se plantea la pregunta, ¿de qué manera esta dependencia -de lo social sobre lo económico- es moderada y no solamente gestionada? Aquí llaman la atención dos aspectos: el primero se dirige particularmente hacia el tema sustentabilidad y qué tan fuerte se entiende la pregunta por el "Soziale Stadt" como una política de distribución para la orientación de recursos. Si se quisiese profundizar en el enfoque del "Capital Social" de las teorías de Gobernanza, es necesario para un desarrollo sustentable del barrio alejarse de la idea de que esto es solo factible en relación a la situación financiera actual, ya entendida como sustentable, lo cual es especialmente cierto para los proyectos orientados comunitariamente. Queda entonces abierta la pregunta, ipor qué no se desarrolla como una fortaleza una estrategia de empoderamiento, la cual permita el aprovechamiento del capital social a largo plazo? ¿Puede ser que una programática que aún se encuentra en la lógica top-down (grupo de dirección-Management del barrio; finanzas/ poder versus personas/costos) no se encuentre capacitada desde un principio para aspirar a aquella sustentabilidad? Segundo, las discusiones sobre la sustentabilidad señalan el reducido perímetro en que están anclados los proyectos de política comunal. Una de las razones de este bajo anclaje es la convicción fundamental de que el trabajo estaría completamente realizado cuando el Gobierno Fe- 
deral aporta el financiamiento y los proyectos son organizados en la fase preparatoria por el grupo de dirección; con posterioridad ambas instancias se retiran, y es responsabilidad de la localidad "ver como seguir". Las intenciones del "Soziale Stadt" contradicen lo anterior y entienden que el cumplimiento arduo y costoso del trabajo cotidiano del que se ocupan las diferentes oficinas, debiera estar apoyado por un programa adicional; no obstante, para efectos prácticos, sería mejor que este no estuviese integrado a las actividades comunales.

El programa federal no tiene en su perspectiva representaciones más amplias, las cuales debieran estar dirigidas de tal modo que se motive de una forma proactiva a las ciudades participantes en el devenir de los proyectos. Lo anterior en el sentido de una modernización de la administración hacia un cambio estructural y sustentable de su "gobernanza social". Solo de esta forma, conforme al enfoque de la gobernanza, se logra una sustentabilidad organizacional en cada estructura política y administrativa, la cual otorga un lugar fijo a los temas sociales de largo plazo en la política comunal. En los casos en que se ha tenido éxito en la protección de un proyecto o de un barrio gracias a la intervención de un político, devienen aspectos de la política partidaria a la discusión. Por esta razón el anclaje de los proyectos del "Soziale Stadt" a la política comunal los hace correr peligro, ya que la sustentabilidad en cuestión se ve perjudicada cuando a través de las elecciones comunales se instalan nuevas condiciones de las mayoría. Poner la problemática del "Soziale Stadt" en la cúspide de los temas diarios de la política comunal en algunos casos tuvo éxito gracias al prominente compromiso que adquieren actores respetados o institucionalmente acreditados. El anclaje sustentable de la problemática requiere de un enfoque más amplio en la política comunal, el cual incremente la dirección administrativa interna y la estructuración de los actores en nuevas redes.

Los límites de la sustentabilidad del programa federal en su forma organizacional están estructuralmente en conexión con la alta organización política. Con esto son mencionados como factores inhibidores sobre todo los que hacen referencia a la viabilidad del programa. Como inapropiado se percibe que los procesos a largo plazo se entiendan como un objetivo pero se definan en un horizonte de dirección temporalmente acotado. Las obligaciones de los niveles locales y supralocales son entendidas así como periódicas. De esto deriva un efecto negativo:

"Por un par de años todo es lindo, después caeremos nuevamente en la situación de antes. Así uno no puede relacionarse con las personas. Cuando se quiere motivar a la gente a cuidar su propio barrio duraderamente, no es una sorpresa que todos en algún momento estén hartos (...) de proyecto a proyecto nadie quiere dejarse entretener con promesas que no se cumplen" (entrevista $\mathrm{N}^{\circ} 16$ ) 
El anclaje temporal del programa en el sistema federal es gracias a una filosofía de diseño político; con esta se ha iniciado una reforma orientada contra la rigidez de la norma jurídica, apoyada argumentativamente por la NPM, que entrega progresivos puntos de partida para la acción en muchas áreas de la política. No obstante, en relación a la pregunta de si la tarea política básica de la sociedad es organizar la sustentabilidad social de las ciudades como un "proyecto", deja bastantes dudas.

El error organizacional del programa federal "Soziale Stadt" consiste en la conexión que tiene con el fomento urbano, cuando su objetivo es lograr la sustentabilidad de la cohesión social en las ciudades. En esta perspectiva organizacional es posible encontrar dinámicas particulares, las cuales se vuelven perceptibles cuando se pasa desde el diseño de los proyectos a su implementación en el lugar. Aunque es opcional para las ciudades si estas actúan fuertemente en temas urbanos o más bien se orientan a temas comunitarios, lo que normalmente ocurre es la realización de proyectos combinando ambos aspectos. Planeadores urbanos y arquitectos cumplen en muchos de estos proyectos un rol importante, tomando parte en funciones de dirección, en el contacto con otras oficinas y con actores de distintas redes sociales. La vinculación entre las competencias necesarias para las inversiones en infraestructura y diseño con las tareas de dirección resultan de una lógica de sujeción superior. Sin embargo estas personas no cuentan con una educación o preparación en las tareas que requiere la dirección, limitándose así la mayoría de las veces su potencial argumentativo a un análisis técnico de factibilidad dentro de su campo, esto es el desarrollo urbano y el desarrollo de proyectos de construcción.

\section{"Local Governance" für die "Soziale Stadt"?}

Como se puede analizar en observaciones realizadas de manera preeliminar y selectiva, la práctica del programa federal "Soziale Stadt" se orienta de manera muy vaga a las necesidades y especificaciones de un cambio político de orden superior, como es la "modernización del Estado". El programa "Soziale Stadt" responde a una filosofía que se basa en las oportunidades que genera un proyecto limitado temporalmente. Con esto como programática se buscan las ventajas de una activación del potencial local, aun cuando se toca solo de manera tangencial la autonomía de las ciudades en el diseño del desarrollo social. Esta perspectiva se debe observar con mucha cautela. No obstante las prácticas del "Soziale Stadt" estimulan la dirección de los programas hacia ciertos temas y fomentan además, con el apoyo del grupo de dirección y del management del barrio, innovaciones en la organización administrativa. Esta oportunidad de trans- 
formación, que sería necesaria para un desarrollo urbano social con base en "gobernanza local", no podrían ser utilizadas por las ciudades en otro sentido. El hincapié en el nivel "menor" de dirección posibilita el fortalecimiento de las redes locales y de un enfoque de empoderamiento, lo cual hasta ahora no se incluía organizacionalmente en la política de las ciudades.

A pesar del necesario reconocimiento del capital social existente en los barrios, la acción política dirigida a introducir una gobernanza local orientada socialmente es visiblemente débil. En el diseño básico de la política comunal, las tareas en conjunto, como es la "jerarquización top-down", transforma solo de manera moderada los instrumentos de dirección ya existentes, sin embargo cabe destacar que estas transformaciones no son posibles de aplicar completamente. Por otro lado, allí donde las influencias de los barrios y de los proyectos individuales sobre la política y la administración comunal estaban presentes, se observa un fortalecimiento de la gobernanza orientada socialmente. Sin embargo, para esto las redes extensas no son suficientes, puesto que el anclaje del poder político y el consenso comunal a través de una amplia participación ciudadana pueden lograr que se establezca un desarrollo de las ciudades socialmente duradero, por sobre otras obligaciones que impone la gobernanza (como por ejemplo la seguridad pública). La sustentabilidad de los proyectos individuales, la cual se persigue en el marco del programa federal, puede contribuir a una reforma estratégica de esta envergadura. Sin embargo, esta puede ser también un factor de interferencia, debido a su disminuida incorporación en una política comunal, cuya reforma administrativa solo tiene como fin un aumento de la eficiencia en la rutina diaria.

\section{Referencias bibliográficas}

ATKINSON, Rob y ECKARDT, Frank. Urban regeneration policies in Europe. En: ECKARDT, Frank y KREISL, Peter (eds.). City images and urban regeneration. Frankfurt am Main, Peter Lang. 2003. p. 33-66. ISBN 978-3-631-50701-8.

BERNT, Matthias y PRITSCHE, Miriam. Von programmen zu projekten: Die ambivalenten innovationen des quartiersmanagements. En: GREIFFENHAGEN, Sylvia y NELLER, Katja (eds.) Praxis ohne theorie? Wissenschaftliche Diskurse zum Bund-Länder-Programm Stadtteile mit besonderem Entwicklungsbedarf - Die soziale Stadt. Wiesbaden, VS-Verlag. 2005. p. 219-236. ISBN 3-8100-4202-1.

BOGASON, Peter. Public policy and local governance institutions in postmodern society. Cheltenham UK, Edward Elgar. 2000. 200 p. ISBN 840-64891-0.

BOIVARD, Tony, LÖFFLER, Elke y PARRADO-DIEZ, Salvador. Emerging practices in network management at local levels in Europe. En: BOIVARD, 
Tony, LÖFFLER, Elke y PARRADO-DIEZ, Salvador (eds.) Developing local governance in networks in Europe. Baden-Baden, Nomos Verlagsgesellschaft. 2002. p.9-24. ISBN 3-7890-7826-3.

DEUTSCHES Institut Frir Urbanistik DIFU (ed). Die Soziale Stadt. Eine erste Bilanz des Bund-LänderProgramms "Stadtteile mit besonderem Entwicklungsbedarf - die Soziale Stadt". Berlin, DIFU. 2002.

Programmgrundlagen. Arbeitspapiere zum Programm Soziale Stadt 3. Berlin, DIFU. 2000.

LAHNER, Marion y ZIMMERMANN, Karsten. Integrierte Stadtteilentwicklung: Bedeutung, Zusammenhang und Grenzen von Place-making, Sozialkapital und neuen Formen der Local Governance. En: GREIFFENHAGEN, Sylvia y NELLER, Katja (Ed) Praxis ohne Theorie? Wissenschaftliche Diskurse zum Bund-Länder-Programm Stadtteile mit besonderem Entwicklungsbedarf Die soziale Stadt. Wiesbaden, VS-Verlag. 2005. p. 219-236. ISBN 3-8100-4202-1.

LE GALÈS, Patrick. European cities. Social conflicts and governance. New York, Oxford University Press. 2002. 328 p. ISBN 0-19-92-4357-3.
MINGUE, Martin, POLIDANO, Charles, HULME, David, ELGAR, Edward. Beyond the new public management. Changing ideas and practices in governance. Cheltenham UK, Edward Elgar. 1998. 308 p. ISBN 1-8589-8913-2.

NASCHOLD, Frieder, BOGUMIL, Jörg. Modernisierung des Staates. New public management aus deutscher und internationaler perspektive. Opladen, Leske+Budrich. 2000. 251 p. ISBN: 3-8100-28487.

SCHADER-STIFTUNG (ed). Politische Steuerung der Stadtentwicklung. Das Programm, "Die soziale Stadt" in der Diskussion. Darmstadt, SchaderStiftung. 2001.

WALTHER, Uwe-Jens. Ambitionen und Ambivalenzen eines Programms. Die Soziale Stadt zwischen neuen Herausforderungen und alten Lösungen. En WALTHER, Uwe-Jens (ed). Soziale StadtZwischenbilanzen. Ein Programm auf dem Weg zur "Sozialen Stadt"? Opladen, Leske+Budrich. 2002. p. 23-44. ISBN 3-8100-3592-0.

WENDLAND, Anja. Mitbestimmung oder Beteiligung im Laufstall? Zur Diskussion um Partizipation in der sozialen Stadtteilentwicklung. Bielefeld, Kleine Verlag. 2002. 141 p. ISBN 3-89370-366-7. 